\title{
On the Moments and the Distribution of the Cost of a Semi Markov Model for Healthcare Systems
}

\author{
Aleka A. Papadopoulou • George Tsaklidis • \\ Sally McClean · Lalit Garg
}

Received: 1 February 2011 / Revised: 6 October 2011 /

Accepted: 10 October 2011 / Published online: 30 October 2011

(C) Springer Science+Business Media, LLC 2011

\begin{abstract}
In this paper we extend our previous semi-Markov reward model which attached costs to duration in states, by including costs of making a transition from one state to another. Theoretical results concerning the moments and consequently the distribution of interval costs for every member and of the total cost per unit period at any time and also through time intervals are obtained and provided in analytic form for the semi Markov reward model with discounting. The results are applied to an open healthcare system. In the healthcare domain such transition costs allow us to evaluate the overall costs of therapy or clinical intervention where an operation or other treatment may be an option. This model can be used for strategic approaches to planning and evaluating long-term patient care. The results demonstrate the potential of the model to demonstrate differential costs of different therapeutic strategies and explore optimal solutions.
\end{abstract}

Keywords Healthcare $\cdot$ Semi-Markov systems $\cdot$ Cost

AMS Subject Classifications (2000) $60 \mathrm{~K} 15 \cdot 60 \mathrm{~K} 20 \cdot 60 \mathrm{~J} 20$

\footnotetext{
A. A. Papadopoulou $(\varangle) \cdot$ G. Tsaklidis

Department of Mathematics, Aristotle University of Thessaloniki,

Thessaloniki 54124, Greece

e-mail: apapado@math.auth.gr

G. Tsaklidis

e-mail: tsaklidi@math.auth.gr

S. McClean · L. Garg

School of Computing and Information Engineering, University of Ulster, BT52 1SA, Coleraine, Northern Ireland, UK

S. McClean

e-mail: si.mcclean@ulster.ac.uk

L. Garg

e-mail: L.Garg@ulster.ac.uk
} 


\section{Introduction}

We have previously used semi-Markov systems to describe movements of individuals between states (McClean et al. 2004, 2008; Papadopoulou 2001, 2004, 2010; Vassiliou and Papadopoulou 1992). The movements may be actual or virtual and described by a phase-type distribution (McClean and Millard 2006). This model can be extended to a semi Markov reward model (Howard 1971) for open and closed systems, with Poisson admissions and an absorbing state. The distribution of costs at any time and in a given time interval were also previously evaluated and expressions found for the corresponding means and variances. In literature, basic definitions and theoretical results for the homogeneous semi-Markov reward processes can be found in Howard (1971). De Dominicis and Manca (1986) provided the first results on the transient behaviour of the semi-Markov reward processes and applied them to insurance disability problems. In Balcer and Sahin (1986) two extensions of a semi-Markov reward model of pension accumulation are examined and expressions for the mean expected benefits are derived. A multivariate reward process defined on a semiMarkov process is studied in Masuda and Sumita (1991) and transform results for the distributions of the multivariate reward processes are derived. In Masuda (1993) partially observable semi-Markov reward processes are examined and the conditional distribution of the vector with total rewards is studied. A general definition of rewards can be found in Limnios and Oprisan (2001) and the study of the asymptotic behaviour of semi-Markov reward process in Reza Soltani and Khorshidian (1998). Later, in Papadopoulou (2004) closed analytic forms for the main formulas of the expected reward that the semi Markov system generates are provided. In Jianyong and Xiaobo (2004) average reward semi-Markov decision processes with multichain structure are examined. Also, McClean et al. (2004) provides formulas for semi Markov rewards by means of probability generating functions. In Papadopoulou and Tsaklidis (2007) reward paths for semi Markov models with stochastic selection of the transition probabilities are studied. Furthermore, transition rewards are studied in Janssen and Manca (2007) and higher order moments and variance for semi-Markov rewards are treated numerically in Stenberg et al. (2006) for the homogeneous case and Stenberg et al. (2007) for the non-homogeneous case.

In this paper we have extended the previous semi-Markov reward model to include costs of making a transition from one state to another. Thus in the healthcare domain, for example, we may evaluate the overall costs of therapy or a clinical intervention by adding additional costs and states. This model can be used to determine costs for the entire system for different strategies thus facilitating a systems approach to planning and a holistic approach to costing. For the healthcare domain such models can help us to assess the complex relationship between hospital and community care where there may be possible trade-offs between hospital treatment costs and community care costs. An advantage of our approach is that it allows us to compare different strategies for groups of patients moving through hospital and community care. Stroke disease is particularly amenable to such an approach as patients that do not receive appropriate therapy or rehabilitation in a timely manner may subsequently build up huge costs over time within Social Services in nursing homes and other care facilities. Modeling can assess where and how stroke patients should be treated. In particular, thrombolysis (clot busting drugs), if administered at the right time can produce substantial improvements, both in terms 
of cost and quality of life, by preventing patients from incurring longer term costs in the community.

In this paper we extend our previous semi-Markov reward model to an open system that includes costs of making a transition from one state to another, as well as occupancy costs. This model can be used for strategic approaches to planning and evaluating long-term patient care. The results demonstrate the potential of the models to incorporate differential costs of different therapeutic strategies and explore optimal solutions. In Section 2 we provide theoretical results concerning the moments and consequently the distribution of interval costs for every member of the system and of the total cost per unit period at any time and also through time intervals are obtained and provided in analytic form for the semi Markov reward model with discounting. In Section 3 an example application of the model in the healthcare domain is presented. The model and the results of Section 2 are used for costing thrombolysis services within a hospital. Finally, in Section 4 conclusions for the theory and the application of the present are provided.

\section{Moments and Distribution of the Semi Markov Cost Model with Discounting}

Let us consider a discrete time semi Markov system with finite state space $S=$ $\{1,2, \ldots, k\}$ and the embedded chain defined by the transition probability matrix $\mathbf{P}=$ $\left\{p_{i j}\right\}_{i, j \in S}$ and the holding time mass function matrix $\mathbf{H}(m)=\left\{h_{i j}(m)\right\}_{i, j \in S}$. Holding time mass functions $h_{i j}(m)$ express the probabilities that a member that entered state $i$ at the last transition holds $m$ time units in $i$ before the next transition, given that state $j$ has been selected. Let also $k_{i j}$ be the cost of making a transition from state $i$ to $j$ and $c_{i}$ the cost for occupying state $i$ during a time interval of length 1 . Now, if $\beta$ denotes a discount factor, then the present value of a unit sum paid $n$ time units in the future equals $\beta^{n}, 0<\beta \leq 1$ (Howard 1971). Thus, the present value of cost produced by a member of the system who entered state $i$ at time $s$ and decided to make a transition to state $j$ after $m$ time units in state $i$, equals $(1-\beta)^{-1} \beta^{s}\left(1-\beta^{m}\right) c_{i}+\beta^{s+m} k_{i j}$. In paragraph 2.1 we provide for every $r$ the analytic form of the present value of the $r$-th moments of cost spent by the system for a member through a time interval. In paragraphs 2.2 and 2.3 we provide results concerning the present value of the moments of the total cost for the system for one time unit interval and also for the interval $(0, n)$.

\subsection{Moments of Interval Cost for the Chain with Discounting}

The Mean Costs Let us now define by $T_{t, n, \beta}^{i}$ the present value of cost spent by the system for a member until time $n$ given that the member is recruited at time $t$ to state $i$ and the discount factor is $\beta$. Let also $v_{i}(t, n, \beta)$ be the expected value of $T_{t, n, \beta}^{i}$, i.e., $v_{i}(t, n, \beta)=E\left(T_{t, n, \beta}^{i}\right)$ and $\mathbf{v}(t, n, \beta)=\left[v_{1}(t, n, \beta), \ldots, v_{k}(t, n, \beta)\right]$ t the vector of the expected present values of cost through the interval $(t, n]$, with $\mathbf{v}(n, n, \beta)=\mathbf{0}$. In the following theorem a closed analytic form in relation with the basic parameters is provided for the vector $\mathbf{v}(t, n, \beta)$. 
Theorem 2.1 The vector $\mathbf{v}(t, n, \beta)$ of the present value of the expected cost through the interval $(t, n]$ is described by the following equation

$$
\begin{aligned}
\mathbf{v}(t, n, \beta)= & \left(\mathbf{G}(n-t) \mathbf{1}^{\prime}\right) \diamond \mathbf{c}_{1}(t, n, \beta)+\mathbf{b}_{1}(t, n-t, \beta) \\
& +\sum_{j=2}^{n-t}[\mathbf{E}(j-1)]\left[\left(\mathbf{G}(n-t-j+1) \mathbf{1}^{\prime}\right) \diamond \mathbf{c}_{1}(t+j-1, n, \beta)\right. \\
& \left.+\mathbf{b}_{1}(t+j-1, n-t-j+1, \beta)\right]
\end{aligned}
$$

where

$$
\begin{aligned}
& \mathbf{G}(n)=\operatorname{diag}\left\{\sum_{j=1}^{k} p_{1 j} \sum_{m=n+1}^{\infty} h_{1 j}(m), \ldots, \sum_{j=1}^{k} p_{k j} \sum_{m=n+1}^{\infty} h_{k j}(m)\right\}, \\
& \mathbf{c}_{1}(t, n, \beta)=\left[(1-\beta)^{-1}\left(\beta^{t}-\beta^{n}\right) c_{1}, \ldots,(1-\beta)^{-1}\left(\beta^{t}-\beta^{n}\right) c_{k}\right]^{\prime},
\end{aligned}
$$

$\diamond$ denotes the Hadanard (elementwise) product,

$$
\begin{aligned}
\mathbf{b}_{1}(t, n, \beta) & =\sum_{m=1}^{n}\left[[\mathbf{P} \diamond \mathbf{H}(m)] \diamond \mathbf{C K}_{1}(t, m, \beta)\right] \mathbf{1}^{\prime} \\
& =\left\{\sum_{j=1}^{k} p_{i j} \sum_{m=1}^{n} h_{i j}(m)\left[(1-\beta)^{-1} \beta^{t}\left(1-\beta^{m}\right) c_{i}+\beta^{t+m} k_{i j}\right]\right\}_{i=1,2, \ldots k},
\end{aligned}
$$

and $\mathbf{E}(n)=\left\{e_{i j}(n)\right\}_{i, j \in S}$ stands for the matrix of the entrance probabilities to a state after time $n$, whose $\mathbf{E}(n)$ analytic form is:

$$
\begin{aligned}
\mathbf{E}(n)= & {[\mathbf{P} \diamond \mathbf{H}(n)]+\sum_{j=2}^{n}[\mathbf{P} \diamond \mathbf{H}(j-1)][\mathbf{P} \diamond \mathbf{H}(n-j+1)] } \\
& +\sum_{j=2}^{n} \sum_{x=1}^{j-2} \mathbf{S}_{j}\left(x, m_{x}\right)[\mathbf{P} \diamond \mathbf{H}(n-j+1)],
\end{aligned}
$$

where

$$
\mathbf{E}(0)=\mathbf{I}, \mathbf{S}_{j}\left(x, m_{x}\right)=\sum_{m_{x}=2}^{j-x} \sum_{m_{x-1}=1+m_{x}}^{j-x+1} \cdots \sum_{m_{1}=1+m_{2}}^{j-1} \prod_{r=-1}^{x-1}\left[\mathbf{P} \diamond \mathbf{H}\left(m_{x-r-1}-m_{x-r}\right)\right]
$$

if $j \geq x+2$, and $\mathbf{S}_{j}\left(x, m_{x}\right)=\mathbf{0}$, if $j<x+2$.

Proof Using probabilistic argument we derive the following recursive equation

$$
\begin{aligned}
v_{i}(t, n, \beta)= & \sum_{j=1}^{k} p_{i j} \sum_{m=n+1-t}^{\infty} h_{i j}(m)(1-\beta)^{-1}\left(\beta^{t}-\beta^{n}\right) c_{i} \\
& +\sum_{j=1}^{k} p_{i j} \sum_{m=1}^{n-t} h_{i j}(m)\left[(1-\beta)^{-1} \beta^{t}\left(1-\beta^{m}\right) c_{i}+\beta^{t+m} k_{i j}\right] \\
& +\sum_{j=1}^{k} p_{i j} \sum_{m=1}^{n-t} h_{i j}(m) v_{j}(t+m, n, \beta) .
\end{aligned}
$$


In matrix notation the previous equation becomes

$$
\mathbf{v}(t, n, \beta)=\left(\mathbf{G}(n-t) \mathbf{1}^{\prime}\right) \diamond \mathbf{c}_{1}(t, n, \beta)+\mathbf{b}_{1}(t, n-t, \beta)+\sum_{m=1}^{n-t}[\mathbf{P} \diamond \mathbf{H}(m)] \mathbf{v}(t+m, n, \beta) .
$$

From Eq. 2.1 by applying a similar methodology with that of Papadopoulou (2004) we can derive the vector of the mean costs through the interval $(t, n]$ for every member in closed analytic form which is given in Eq. 2.1.

\section{The Expected Squared Costs Define as}

$v_{i}^{2}(t, n, \beta)=[$ the expected present value of the squared cost for $(t, n]$ given that a member entered state iat time $\mathrm{t}$ and the discount factor is $\beta$ ].

Now we define the following matrices:

$\mathbf{C K}_{x}(t, m, \beta)$ is a $k \times k$ matrix, $\mathbf{C K}_{x}(t, m, \beta)=\left\{\left((1-\beta)^{-1} \beta^{t}\left(1-\beta^{m}\right) c_{i}+\beta^{t+m} k_{i j}\right)^{x}\right\}$ for every $x=1,2, \ldots$, and $\mathbf{C K}_{0}(m)=\mathbf{U}, \mathbf{c}_{x}(t, n, \beta)$ is a $k \times 1$ vector, where $\mathbf{c}_{x}(t, n, \beta)=$ $\left\{\mathrm{c}_{i}^{x}\left[(1-\beta)^{-1}\left(\beta^{t}-\beta^{n}\right)\right]^{x}\right\}$ for every $x=1,2, \ldots, \mathbf{v}^{x}(t, n, \beta)=\left\{v_{i}^{x}(t, n, \beta)\right\}$ is a $k \times 1$ vector where $v_{i}^{x}(t, n, \beta)$ is equal to the present value of the $x$-th moment of cost until time $n$ given that a member entered state $i$ at time $t$, for every $x=2,3, \ldots, \mathbf{b}_{x}(t, n, \beta)$, $x=1,2, \ldots$, is a $k \times 1$ vector such that,

$$
\begin{aligned}
\mathbf{b}_{x}(t, n, \beta) & =\sum_{m=1}^{n}\left[[\mathbf{P} \diamond \mathbf{H}(m)] \diamond \mathbf{C K}_{x}(t, m, \beta)\right] \mathbf{1}^{\prime} \\
& =\left\{\sum_{j=1}^{k} p_{i j} \sum_{m=1}^{n} h_{i j}(m)\left[(1-\beta)^{-1} \beta^{t}\left(1-\beta^{m}\right) c_{i}+\beta^{t+m} k_{i j}\right]^{x}\right\}_{i=1,2, \ldots k} .
\end{aligned}
$$

Thus, if we denote by $\mathbf{v}^{2}(t, n, \beta)=\left[v_{1}^{2}(t, n, \beta), \ldots, v_{k}^{2}(t, n, \beta)\right]^{\prime}$ the vector of the present value of the expected squared costs we can provide by the following theorem a closed analytic form for $\mathbf{v}^{2}(t, n, \beta)$ in relation with its basic parameters.

Theorem 2.2 The vector of the present value of the expected squared costs for the interval $(t, n]$ is given by the equation

$$
\mathbf{v}^{2}(t, n, \beta)=\mathbf{D}(t, n, \beta)+\sum_{j=2}^{n-t}[\mathbf{E}(j-1)][(\mathbf{D}(t+j-1, n, \beta)],
$$

where

$$
\begin{aligned}
& \mathbf{D}(t, n, \beta)=\left(\mathbf{G}(n-t) \mathbf{1}^{\prime}\right) \diamond \mathbf{c}_{2}(t, n, \beta)+\mathbf{b}_{2}(t, n-t, \beta)+2 \sum_{m=1}^{n-t}\left[[\mathbf{P} \diamond \mathbf{H}(m)] \diamond \mathbf{C} \mathbf{K}_{1}(t, m, \beta)\right] \\
& \times\left[\left(\mathbf{G}(n-t-m) \mathbf{1}^{\prime}\right) \diamond \mathbf{c}_{1}(t+m, n, \beta)+\mathbf{b}_{1}(t+m, n-t-m, \beta)\right. \\
& +\sum_{j=2}^{n-t-m}[\mathbf{E}(j-1)]\left[\left(\mathbf{G}(n-t-m-j+1) \mathbf{1}^{\prime}\right) \diamond \mathbf{c}_{1}(t+m+j-1, n, \beta)\right. \\
& \left.\left.+\mathbf{b}_{1}(t+m+j-1, n-t-m-j+1, \beta)\right]\right] .
\end{aligned}
$$


Proof Using probabilistic argument we can result to the following recursive equation

$$
\begin{aligned}
& v_{i}^{2}(t, n, \beta)=E\left[\left(T_{t, n, \beta}^{i}\right)^{2}\right] \\
& =\sum_{j=1}^{k} p_{i j} \sum_{m=n+1-t}^{\infty} h_{i j}(m)\left[(1-\beta)^{-1}\left(\beta^{t}-\beta^{n}\right)\right]^{2} c_{i}^{2} \\
& +\sum_{j=1}^{k} p_{i j} \sum_{m=1}^{n-t} h_{i j}(m) E\left[\left((1-\beta)^{-1} \beta^{t}\left(1-\beta^{m}\right) c_{i}+\beta^{t+m} k_{i j}+T_{t+m, n}^{j}\right)^{2}\right] \\
& =\sum_{j=1}^{k} p_{i j} \sum_{m=n+1-t}^{\infty} h_{i j}(m)\left[(1-\beta)^{-1}\left(\beta^{t}-\beta^{n}\right)\right]^{2} c_{i}^{2} \\
& +\sum_{j=1}^{k} p_{i j} \sum_{m=1}^{n-t} h_{i j}(m) E\left[\left[(1-\beta)^{-1} \beta^{t}\left(1-\beta^{m}\right) c_{i}+\beta^{t+m} k_{i j}\right]^{2}\right] \\
& +\sum_{j=1}^{k} p_{i j} \sum_{m=1}^{n-t} h_{i j}(m) E\left[2\left[(1-\beta)^{-1} \beta^{t}\left(1-\beta^{m}\right) c_{i}+\beta^{t+m} k_{i j}\right] T_{t+m, n, \beta}^{j}\right] \\
& +\sum_{j=1}^{k} p_{i j} \sum_{m=1}^{n-t} h_{i j}(m) E\left[\left[T_{t+m, n, \beta}^{j}\right]^{2}\right] \\
& =\sum_{j=1}^{k} p_{i j} \sum_{m=n+1-t}^{\infty} h_{i j}(m)\left[(1-\beta)^{-1}\left(\beta^{t}-\beta^{n}\right)\right]^{2} c_{i}^{2} \\
& +\sum_{j=1}^{k} p_{i j} \sum_{m=1}^{n-t} h_{i j}(m)\left[(1-\beta)^{-1} \beta^{t}\left(1-\beta^{m}\right) c_{i}+\beta^{t+m} k_{i j}\right]^{2} \\
& +2 \sum_{j=1}^{k} p_{i j} \sum_{m=1}^{n-t} h_{i j}(m)\left[(1-\beta)^{-1} \beta^{t}\left(1-\beta^{m}\right) c_{i}+\beta^{t+m} k_{i j}\right] v_{j}(t+m, n, \beta) \\
& +\sum_{j=1}^{k} p_{i j} \sum_{m=1}^{n-t} h_{i j}(m) v_{j}^{2}(t+m, n, \beta) .
\end{aligned}
$$

Then, using the previous definitions we can derive Eq. 2.4 in matrix notation as follows

$$
\begin{aligned}
\mathbf{v}^{2}(t, n, \beta)= & \left(\mathbf{G}(n-t) \mathbf{1}^{\prime}\right) \diamond \mathbf{c}_{2}(n, t, \beta)+\mathbf{b}_{2}(t, n-t, \beta) \\
& +2 \sum_{m=1}^{n-t}\left[[\mathbf{P} \diamond \mathbf{H}(m)] \diamond \mathbf{C K}_{1}(t, m, \beta)\right] \mathbf{v}(t+m, n, \beta) \\
& +\sum_{m=1}^{n-t}[[\mathbf{P} \diamond \mathbf{H}(m)]] \mathbf{v}^{2}(t+m, n, \beta),
\end{aligned}
$$

with initial condition $\mathbf{v}^{2}(n, n, \beta)=\mathbf{0}$. 
Equation 2.5 expresses recursively the expected squared costs for the interval $(t, n]$. Now, by applying the result of Theorem 2.1 in relation with Eq. 2.5 and following a similar technique with that of Papadopoulou (2004) we can prove Eq. 2.3

Moments of Costs Similarly we define the present values of the $r$-th moments of cost as follows

$\mathbf{v}^{r}(t, n, \beta)=\left\{v_{i}^{r}(t, n, \beta)\right\}_{i}$,

$v_{i}^{r}(t, n, \beta)=[$ the present value of the $\mathrm{r}$-th moment of the cost of a member for the interval $(t, n]$ given that the member entered state $i$ at time $t]$.

Then $v_{i}^{r}(t, n, \beta)=E\left[\left(T_{t, n, \beta}^{i}\right)^{r}\right]$, and thus we have:

Theorem 2.3 The vector of the present values of the r-th moments of the cost for the interval $(t, n]$ is given by the following equation

$$
\mathbf{v}^{r}(t, n, \beta)=\mathbf{A}(r, t, n, \beta)+\sum_{j=2}^{n-t}[\mathbf{E}(j-1)][(\mathbf{A}(r, t+j-1, n, \beta)],
$$

where

$$
\begin{aligned}
\mathbf{A}(r, t, n, \beta)= & \left(\mathbf{G}(n-t) \mathbf{1}^{\prime}\right) \diamond \mathbf{c}_{r}(n, t, \beta)+\mathbf{b}_{r}(t, n-t, \beta) \\
& +\sum_{x=1}^{r-1}\left(\begin{array}{c}
r \\
x
\end{array}\right) \sum_{m=1}^{n-t}\left[[\mathbf{P} \diamond \mathbf{H}(m)] \diamond\left[\mathbf{C} \mathbf{K}_{x}(t, m, \beta)\right]\right] \mathbf{v}^{r-x}(t+m, n, \beta),
\end{aligned}
$$

with initial condition $\mathbf{v}^{x}(n, n, \beta)=\mathbf{0}$.

Proof If we use probabilistic argument we get

$$
\begin{aligned}
v_{i}^{r}(t, n, \beta)= & \sum_{j=1}^{k} p_{i j} \sum_{m=n+1-t}^{\infty} h_{i j}(m)\left[(1-\beta)^{-1}\left(\beta^{t}-\beta^{n}\right)\right]^{r} c_{i}^{r} \\
& +\sum_{j=1}^{k} p_{i j} \sum_{m=1}^{n-t} h_{i j}(m) E\left[\left((1-\beta)^{-1} \beta^{t}\left(1-\beta^{m}\right) c_{i}+\beta^{t+m} k_{i j}+T_{t+m, n, \beta}^{j}\right)^{r}\right] \\
= & \sum_{j=1}^{k} p_{i j} \sum_{m=n+1-t}^{\infty} h_{i j}(m)\left[(1-\beta)^{-1}\left(\beta^{t}-\beta^{n}\right)\right]^{r} c_{i}^{r} \\
& +\sum_{j=1}^{k} p_{i j} \sum_{m=1}^{n-t} h_{i j}(m)\left[\sum_{x=0}^{r-1}\left(\begin{array}{l}
r \\
x
\end{array}\right)\left[(1-\beta)^{-1} \beta^{t}\left(1-\beta^{m}\right) c_{i}+\beta^{t+m} k_{i j}\right]^{x}\right. \\
& {\left.\left[v_{j}^{r-x}(t+m, n)\right]\right] } \\
& +\sum_{j=1}^{k} p_{i j} \sum_{m=1}^{n-t} h_{i j}(m)\left[(1-\beta)^{-1} \beta^{t}\left(1-\beta^{m}\right) c_{i}+\beta^{t+m} k_{i j}\right]^{r} .
\end{aligned}
$$


Equation 2.7 in matrix notation becomes

$$
\begin{aligned}
\mathbf{v}^{\mathbf{r}}(t, n, \beta)= & \left(\mathbf{G}(n-t) \mathbf{1}^{\prime}\right) \diamond \mathbf{c}_{r}(t, n, \beta) \\
& +\sum_{x=0}^{r-1}\left(\begin{array}{c}
r \\
x
\end{array}\right) \sum_{m=1}^{n-t}\left[[\mathbf{P} \diamond \mathbf{H}(m)] \diamond\left[\mathbf{C K}_{x}(t, m, \beta)\right]\right] \mathbf{v}^{\mathbf{r}-\mathbf{x}}(t+m, n, \beta) \\
& +\mathbf{b}_{r}(t, n-t, \beta),
\end{aligned}
$$

where $\mathbf{v}^{x}(n, n, \beta)=\mathbf{0}$.

Finally, if we define

$$
\begin{aligned}
\mathbf{A}(r, t, n, \beta)= & \left(\mathbf{G}(n-t) \mathbf{1}^{\prime}\right) \diamond \mathbf{c}_{r}(t, n, \beta)+\mathbf{b}_{r}(t, n-t, \beta) \\
& +\sum_{x=1}^{r-1}\left(\begin{array}{c}
r \\
x
\end{array}\right) \sum_{m=1}^{n-t}\left[[\mathbf{P} \diamond \mathbf{H}(m)] \diamond\left[\mathbf{C} \mathbf{K}_{x}(t, m, \beta)\right]\right] \mathbf{v}^{r-x}(t+m, n, \beta)
\end{aligned}
$$

and apply the methodology followed in the previous theorems, Eq. 2.8 can be equivalently written as

$$
\mathbf{v}^{r}(t, n, \beta)=\mathbf{A}(r, t, n, \beta)+\sum_{j=2}^{n-t}[\mathbf{E}(j-1)][(\mathbf{A}(r, t+j-1, n, \beta)] .
$$

The Variances of Costs We define the variances of costs as follows:

$$
\begin{aligned}
\operatorname{var}(t, n, \beta)= & \left\{\operatorname{var}_{i}(t, n, \beta)\right\}_{i}, \\
\operatorname{var}_{i}(t, n, \beta)= & {[\text { the present value of the variance of the cost for the interval }(t, n]} \\
& \text { given that a member entered state } i \text { at time } t] .
\end{aligned}
$$

Then we have

Corollary 2.1 The vector of the variances of the costs through the interval $(t, n]$ is given by the following equation

$$
\operatorname{var}(t, n, \beta)=\mathbf{D}(t, n, \beta)+\sum_{j=2}^{n-t}[\mathbf{E}(j-1)][(\mathbf{D}(t+j-1, n, \beta)]-[\mathbf{v}(t, n, \beta) \diamond \mathbf{v}(t, n, \beta)]
$$

for every $t \leq n$,

where

$$
\begin{aligned}
\mathbf{D}(t, n, \beta)= & \left(\mathbf{G}(n-t) \mathbf{1}^{\prime}\right) \diamond \mathbf{c}_{2}(t, n, \beta)+\mathbf{b}_{2}(t, n-t, \beta) \\
& +2 \sum_{m=1}^{n-t}\left[[\mathbf{P} \diamond \mathbf{H}(m)] \diamond \mathbf{C} \mathbf{K}_{1}(t, m, \beta)\right]\left[\left(\mathbf{G}(n-t-m) \mathbf{1}^{\prime}\right) \diamond \mathbf{c}_{1}(t+m, n, \beta)\right. \\
& +\mathbf{b}_{1}(t+m, n-t-m, \beta)+\sum_{j=2}^{n-t-m}[\mathbf{E}(j-1)]\left[\left(\mathbf{G}(n-t-m-j+1) \mathbf{1}^{\prime}\right)\right. \\
& \left.\left.\diamond \mathbf{c}_{1}(t+m+j-1, n, \beta)+\mathbf{b}_{1}(t+m+j-1, n-t-m-j+1, \beta)\right]\right] .
\end{aligned}
$$


and

$$
\begin{aligned}
\mathbf{v}(t, n, \beta)= & \left(\mathbf{G}(n-t) \mathbf{1}^{\prime}\right) \diamond \mathbf{c}_{1}(t, n, \beta)+\mathbf{b}_{1}(t, n-t, \beta) \\
& +\sum_{j=2}^{n-t}[\mathbf{E}(j-1)]\left[\left(\mathbf{G}(n-t-j+1) \mathbf{1}^{\prime}\right) \diamond \mathbf{c}_{1}(t+j-1, n, \beta)\right. \\
& \left.+\mathbf{b}_{1}(t+j-1, n-t-j+1, \beta)\right] .
\end{aligned}
$$

Proof By definition we have that $\operatorname{var}(t, n, \beta)=\mathbf{v}^{2}(t, n, \beta)-[\mathbf{v}(t, n, \beta) \diamond \mathbf{v}(t, n, \beta)]$. Now, if we apply the results of Theorems 2.1, 2.2 we can derive Eq. 2.9.

The Distribution of Costs It is known that for a discrete random variable $X$ with values $x, x=0,1,2, \ldots, N$, yields

$$
P[X=k]=\frac{1}{k !} \sum_{j=0}^{N-k} \frac{(-1)^{j}}{j !} \mu_{(k+j)},
$$

where $\mu_{(r)}$ stands for the $r$-th factorial moment of $X$, which can be found by means of the moments about zero. Thus, by setting $X=T_{t, n, \beta}^{i}$ the probability function of $T_{t, n, \beta}^{i}$ can be evaluated by means of the factorial moments or equivalently the moments about zero as follows:

$$
P\left[T_{t, n, \beta}^{i}=k\right]=\frac{1}{k !} \sum_{j=0}^{N-k} \frac{(-1)^{j}}{j !} \mu_{(k+j)}^{\beta},
$$

where

$$
\begin{aligned}
\mu_{(k+j)}^{\beta} & =E\left(T_{t, n, \beta}^{i}\right)\left(T_{t, n, \beta}^{i}-1\right) \ldots\left(T_{t, n, \beta}^{i}-k-j+1\right)=\sum_{r=0}^{k+j-1}(-1)^{r} s_{r} E\left(\left(T_{t, n, \beta}^{i}\right)^{k+j-r}\right) \\
& =\sum_{r=0}^{k+j-1}(-1)^{r} s_{r} v_{i}^{k+j-r}(t, n, \beta),
\end{aligned}
$$

with $s_{r}=\sum_{1 \leq j_{1}<j_{2} \ldots<j_{r} \leq k+j} j_{1} \ldots j_{r}, s_{o}=1$.

\subsection{System's Mean and Variance of the Total Cost for One Time Unit Interval with Discounting}

Let us consider an open system with Poisson admissions and let $R_{i}(t)$ be the total number of individuals recruited at time $t$, where $R_{i}(t) \sim \operatorname{Poisson}\left(\lambda_{i}\right)$ for every $i=$ $1,2, . ., k$. We have previously defined as $T_{t, n, \beta}^{i}$ the present value of cost spent by the system for a member until time $n$, given that the member was recruited at time $t$ to state $i$ and as $v_{i}(t, n, \beta)$ the mean of $T_{t, n, \beta}^{i}$. Thus, the present value of cost at time $n-1$ is equal to $T_{t, n, \beta}^{i}-T_{t, n-1, \beta}^{i}$. Then the present value of the total cost spent 
at time $n-1$ for all the members recruited to every state at any time is equal to $\sum_{i=1}^{k} \sum_{t=0}^{n-1} R_{i}(t)\left(T_{t, n, \beta}^{i}-T_{t, n-1, \beta}^{i}\right)$. Now, if we denote $\Lambda=\left[\lambda_{1}, \lambda_{2}, \ldots, \lambda_{k}\right]$, we can calculate the present value of the mean total cost spent at time $n-1$ as follows:

Theorem 2.4 The mean of the total cost for all members of the system at time $n-1$ is equal to $\sum_{t=0}^{n} \boldsymbol{\Lambda}(\mathbf{v}(t, n, \beta)-\mathbf{v}(t, n-1, \beta))$, where $\boldsymbol{\Lambda}=\left[\lambda_{1}, \lambda_{2}, \ldots, \lambda_{k}\right], \mathbf{v}(n, n, \beta)=\mathbf{0}$, and

$$
\begin{aligned}
\mathbf{v}(t, n, \beta)= & \left(\mathbf{G}(n-t) \mathbf{1}^{\prime}\right) \diamond \mathbf{c}_{1}(t, n, \beta)+\mathbf{b}_{1}(t, n-t, \beta) \\
& +\sum_{j=2}^{n-t}[\mathbf{E}(j-1)]\left[\left(\mathbf{G}(n-t-j+1) \mathbf{1}^{\prime}\right) \diamond \mathbf{c}_{1}(t+j-1, n, \beta)\right. \\
& \left.+\mathbf{b}_{1}(t+j-1, n-t-j+1, \beta)\right], \\
\mathbf{G}(n)= & \operatorname{diag}\left\{\sum_{j=1}^{k} p_{1 j} \sum_{m=n+1}^{\infty} h_{1 j}(m), \ldots, \sum_{j=1}^{k} p_{k j} \sum_{m=n+1}^{\infty} h_{k j}(m)\right\} \\
\mathbf{c}_{1}(t, n, \beta)= & {\left[(1-\beta)^{-1}\left(\beta^{t}-\beta^{n}\right) c_{1}, \ldots,(1-\beta)^{-1}\left(\beta^{t}-\beta^{n}\right) c_{k}\right]^{\prime}, } \\
\mathbf{b}_{1}(t, n, \beta)= & \sum_{m=1}^{n}\left[[\mathbf{P} \diamond \mathbf{H}(m)] \diamond \mathbf{C} \mathbf{K}_{1}(t, m, \beta)\right] \mathbf{1}^{\prime} \\
= & \left\{\sum_{j=1}^{k} p_{i j} \sum_{m=1}^{n} h_{i j}(m)\left[(1-\beta)^{-1} \beta^{t}\left(1-\beta^{m}\right) c_{i}+\beta^{t+m} k_{i j}\right]\right.
\end{aligned} .
$$

Proof

$$
\begin{aligned}
E\left(\sum_{i=1}^{k} \sum_{t=0}^{n-1} R_{i}(t)\left(T_{t, n, \beta}^{i}-T_{t, n-1, \beta}^{i}\right)\right) & =E\left(\sum_{i=1}^{k} \sum_{t=0}^{n-1} R_{i}(t)\left(T_{t, n, \beta}^{i}-T_{t, n-1, \beta}^{i}\right)\right) \\
& =\sum_{i=1}^{k} \sum_{t=0}^{n-1} E\left(R_{i}(t)\right) E\left(T_{t, n, \beta}^{i}-T_{t, n-1, \beta}^{i}\right) \\
& =\sum_{i=1}^{k} \sum_{t=0}^{n-1} \lambda_{i}(t)\left(v_{i}(t, n, \beta)-v_{i}(t, n-1, \beta)\right) \\
& =\sum_{t=0}^{n-1} \boldsymbol{\Lambda}(\mathbf{v}(t, n, \beta)-\mathbf{v}(t, n-1, \beta))
\end{aligned}
$$

In the above we will provide an analytic form for the variance of the total cost spent at time $n-1$. 
Theorem 2.5 Let $\mathbf{v v}^{x, r-x}(t, n, \beta)=\left\{v v_{i}^{x, r-x}(t, n, \beta)\right\}_{i}$ be the $k \times 1$ vector, where $v v_{i}^{x, r-x}(t, n, \beta)=E\left(\left(T_{t, n, \beta}^{i}\right)^{x}\left(T_{t, n-1, \beta}^{i}\right)^{r-x}\right)$. Then

$$
\begin{aligned}
& \mathbf{v v}^{x, r-x}(t, n, \beta)=\left(\frac{\beta^{t}-\beta^{n}}{\beta^{t}-\beta^{n-1}}\right)^{x}\left(\mathbf{G}(n-t) \mathbf{1}^{\prime}\right) \diamond \mathbf{c}_{r}(t, n-1, \beta) \\
& +\sum_{z=0}^{x} \sum_{w=0}^{r-x}\left(\begin{array}{l}
x \\
z
\end{array}\right)\left(\begin{array}{c}
r-x \\
w
\end{array}\right) \\
& \times \sum_{m=1}^{n-t-1}\left[[\mathbf{P} \diamond \mathbf{H}(m)] \diamond\left[\mathbf{C K}_{z+w}(t, m, \beta)\right]\right]\left[\mathbf{v v}^{x-z, r-x-w}(t+m, n, \beta)\right] \\
& +\left[\left[[\mathbf{P} \diamond \mathbf{H}(n-t)] \diamond \mathbf{C K}_{x}(t, n-t, \beta)\right] \mathbf{1}^{\prime}\right] \diamond \mathbf{c}_{r-x}(t, n-1, \beta) .
\end{aligned}
$$

Proof Using probabilistic argument we have that

$$
\begin{aligned}
& v v_{i}^{x, r-x}(t, n, \beta)=\sum_{j=1}^{k} p_{i j} \sum_{m=n+1-t}^{\infty} h_{i j}(m)\left[\left((1-\beta)^{-1}\left(\beta^{t}-\beta^{n}\right) c_{i}\right)^{x}\left((1-\beta)^{-1}\left(\beta^{t}-\beta^{n-1}\right) c_{i}\right)^{r-x}\right] \\
& +\sum_{j=1}^{k} p_{i j} \sum_{m=1}^{n-t-1} h_{i j}(m) E\left[\left[(1-\beta)^{-1} \beta^{t}\left(1-\beta^{m}\right) c_{i}+\beta^{t+m} k_{i j}+T_{t+m, n, \beta}^{j}\right]^{x}\right. \\
& \left.\cdot\left[(1-\beta)^{-1} \beta^{t}\left(1-\beta^{m}\right) c_{i}+\beta^{t+m} k_{i j}+T_{t+m, n-1, \beta}^{j}\right]^{r-x}\right] \\
& +\sum_{j=1}^{k} p_{i j} h_{i j}(n-t)\left[(1-\beta)^{-1}\left(\beta^{t}-\beta^{n}\right) c_{i}+\beta^{n} k_{i j}\right]^{x}\left[(1-\beta)^{-1}\left(\beta^{t}-\beta^{n-1}\right) c_{i}\right]^{r-x} \\
& =\sum_{j=1}^{k} p_{i j} \sum_{m=n+1-t}^{\infty} h_{i j}(m)\left[\left(\frac{\beta^{t}-\beta^{n}}{\beta^{t}-\beta^{n-1}}\right)^{x}\left((1-\beta)^{-1}\left(\beta^{t}-\beta^{n-1}\right) c_{i}\right)^{r}\right] \\
& +\sum_{j=1}^{k} p_{i j} \sum_{m=1}^{n-t-1} h_{i j}(m) \\
& \left.\times E\left[\sum_{z=0}^{x}\left(\begin{array}{l}
x \\
z
\end{array}\right)\left[(1-\beta)^{-1} \beta^{t}\left(1-\beta^{m}\right) c_{i}+\beta^{t+m} k_{i j}\right]^{z} T_{t+m, n}^{j}\right]^{x-z}\right] \\
& \cdot\left[\sum_{w=0}^{r-x}\left(\begin{array}{c}
r-x \\
w
\end{array}\right)\left[(1-\beta)^{-1} \beta^{t}\left(1-\beta^{m}\right) c_{i}+\beta^{t+m} k_{i j}\right]^{w}\left[T_{t+m, n-1}^{j}\right]^{r-x-w}\right] \\
& +\sum_{j=1}^{k} p_{i j} h_{i j}(n-t)\left[(1-\beta)^{-1}\left(\beta^{t}-\beta^{n}\right) c_{i}+\beta^{n} k_{i j}\right]^{x}\left[(1-\beta)^{-1}\left(\beta^{t}-\beta^{n-1}\right) c_{i}\right]^{r-x}
\end{aligned}
$$




$$
\begin{aligned}
= & \sum_{j=1}^{k} p_{i j} \sum_{m=n+1-t}^{\infty} h_{i j}(m)\left[\left(\frac{\beta^{t}-\beta^{n}}{\beta^{t}-\beta^{n-1}}\right)^{x}\left((1-\beta)^{-1}\left(\beta^{t}-\beta^{n-1}\right) c_{i}\right)^{r}\right] \\
& +\sum_{z=0}^{x} \sum_{w=0}^{r-x}\left(\begin{array}{c}
x \\
z
\end{array}\right)\left(\begin{array}{c}
r-x \\
w
\end{array}\right) \sum_{j=1}^{k} p_{i j} \sum_{m=1}^{n-t-1} h_{i j}(m) \\
& \times\left[(1-\beta)^{-1} \beta^{t}\left(1-\beta^{m}\right) c_{i}+\beta^{t+m} k_{i j}\right]^{z+w} \cdot E\left[\left[T_{t+m, n, \beta}^{j}\right]^{x-z}\left[T_{t+m, n-1, \beta}^{j}\right]^{r-x-w}\right] \\
& +\sum_{j=1}^{k} p_{i j} h_{i j}(n-t)\left[(1-\beta)^{-1}\left(\beta^{t}-\beta^{n}\right) c_{i}+\beta^{n} k_{i j}\right]^{x}\left[(1-\beta)^{-1}\left(\beta^{t}-\beta^{n-1}\right) c_{i}\right]^{r-x} .
\end{aligned}
$$

The above result in matrix form becomes

$$
\begin{aligned}
\mathbf{v v}^{x, r-x}(t, n, \beta)= & \left(\frac{\beta^{t}-\beta^{n}}{\beta^{t}-\beta^{n-1}}\right)^{x}\left(\mathbf{G}(n-t) \mathbf{1}^{\prime}\right) \diamond \mathbf{c}_{r}(t, n-1, \beta)+\sum_{z=0}^{x} \sum_{w=0}^{r-x}\left(\begin{array}{c}
x \\
z
\end{array}\right)\left(\begin{array}{c}
r-x \\
w
\end{array}\right) \\
& \times \sum_{m=1}^{n-t-1}\left[[\mathbf{P} \diamond \mathbf{H}(m)] \diamond\left[\mathbf{C} \mathbf{K}_{z+w}(t, m, \beta)\right]\right]\left[\mathbf{v} \mathbf{v}^{x-z, r-x-w}(t+m, n, \beta)\right] \\
& +\left[\left[[\mathbf{P} \diamond \mathbf{H}(n-t)] \diamond \mathbf{C} \mathbf{K}_{x}(t, n-t, \beta)\right] \mathbf{1}^{\prime}\right] \diamond \mathbf{c}_{r-x}(t, n-1, \beta) .
\end{aligned}
$$

The initial condition is $\mathbf{v v}^{x, r-x}(n-1, n, \beta)=\mathbf{0}$.

Theorem 2.6 The variance of the total cost for all members of the system at time $n-1$ is given by

$$
\begin{aligned}
V & \operatorname{Vr}\left(\sum_{i=1}^{k} \sum_{t=0}^{n-1} R_{i}(t)\left(T_{t, n, \beta}^{i}-T_{t, n-1, \beta}^{i}\right)\right) \\
& =\sum_{t=0}^{n-1}(\boldsymbol{\Lambda}+(\mathbf{\Lambda} \diamond \mathbf{\Lambda}))\left(\mathbf{v}^{2}(t, n, \beta)+\mathbf{v}^{2}(t, n-1, \beta)-2 \mathbf{v} \mathbf{v}^{1,1}(t, n, \beta)\right) \\
& +\sum_{\left(i, t_{1}\right) \neq\left(j, t_{2}\right)} \lambda_{i} \lambda_{j}\left(v_{i}\left(t_{1}, n, \beta\right)-v_{i}\left(t_{1}, n-1, \beta\right)\right)\left(v_{j}\left(t_{2}, n, \beta\right)-v_{j}\left(t_{2}, n-1, \beta\right)\right) \\
& -\left(\sum_{t=0}^{n-1} \mathbf{\Lambda}(\mathbf{v}(t, n, \beta)-\mathbf{v}(t, n-1, \beta))\right)^{2},
\end{aligned}
$$

where

$$
\begin{aligned}
\mathbf{v v}^{1,1}(t, n, \beta)= & \left(\frac{\beta^{t}-\beta^{n}}{\beta^{t}-\beta^{n-1}}\right)\left(\mathbf{G}(n-t) \mathbf{1}^{\prime}\right) \diamond \mathbf{c}_{2}(t, n-1, \beta) \\
& +\sum_{m=1}^{n-t-1}\left[[\mathbf{P} \diamond \mathbf{H}(m)] \diamond\left[\mathbf{C} \mathbf{K}_{2}(t, m, \beta)\right]\right] \mathbf{1}^{\prime} \\
& +\sum_{m=1}^{n-t-1}\left[[\mathbf{P} \diamond \mathbf{H}(m)] \diamond\left[\mathbf{C} \mathbf{K}_{1}(t, m, \beta)\right]\right][\mathbf{v}(t+m, n, \beta)+\mathbf{v}(t+m, n-1, \beta)] \\
& +\sum_{m=1}^{n-t-1}[\mathbf{P} \diamond \mathbf{H}(m)]\left[\mathbf{v} \mathbf{v}^{1,1}(t+m, n, \beta)\right] \\
& +\left[\left[[\mathbf{P} \diamond \mathbf{H}(n-t)] \diamond \mathbf{C K}_{1}(t, n-t, \beta)\right] \mathbf{1}^{\prime}\right] \diamond \mathbf{c}_{1}(t, n-1, \beta) .
\end{aligned}
$$


Proof The second moment of the total cost is equal to $E\left(\left(\sum_{i=1}^{k} \sum_{t=0}^{n-1} R_{i}(t)\left(T_{t, n, \beta}^{i}-T_{t, n-1, \beta}^{i}\right)\right)^{2}\right)$. After some calculations we result to the following equation

$$
\begin{aligned}
& E\left(\left(\sum_{i=1}^{k} \sum_{t=0}^{n-1} R_{i}(t)\left(T_{t, n, \beta}^{i}-T_{t, n-1, \beta}^{i}\right)\right)^{2}\right) \\
& =\sum_{t=0}^{n-1}(\boldsymbol{\Lambda}+(\mathbf{\Lambda} \boldsymbol{\Lambda}))\left(\mathbf{v}^{2}(t, n, \beta)+\mathbf{v}^{2}(t, n-1, \beta)-2 \mathbf{v v}^{1,1}(t, n, \beta)\right) \\
& \quad+\sum_{\left(i, t_{1}\right) \neq\left(j, t_{2}\right)}^{\lambda_{i} \lambda_{j}}\left(v_{i}\left(t_{1}, n, \beta\right)-v_{i}\left(t_{1}, n-1, \beta\right)\right)\left(v_{j}\left(t_{2}, n, \beta\right)-v_{j}\left(t_{2}, n-1, \beta\right)\right) .
\end{aligned}
$$

If we apply Theorem 2.5 for $\mathrm{r}=2, \mathrm{x}=1$, we can calculate $\mathbf{v v ^ { 1 , 1 }}(t, n, \beta)$ that appears in Eq. 2.10 as follows:

$$
\begin{aligned}
\mathbf{v} \mathbf{v}^{1,1}(t, n, \beta)= & \left(\frac{\beta^{t}-\beta^{n}}{\beta^{t}-\beta^{n-1}}\right)\left(\mathbf{G}(n-t) \mathbf{1}^{\prime}\right) \diamond \mathbf{c}_{2}(t, n-1, \beta) \\
& +\sum_{m=1}^{n-t-1}\left[[\mathbf{P} \diamond \mathbf{H}(m)] \diamond\left[\mathbf{C} \mathbf{K}_{2}(t, m, \beta)\right]\right] \mathbf{1}^{\prime} \\
& +\sum_{m=1}^{n-t-1}\left[[\mathbf{P} \diamond \mathbf{H}(m)] \diamond\left[\mathbf{C} \mathbf{K}_{\mathbf{1}}(t, m, \beta)\right]\right][\mathbf{v}(t+m, n, \beta)+\mathbf{v}(t+m, n-1, \beta)] \\
& +\sum_{m=1}^{n-t-1}[\mathbf{P} \diamond \mathbf{H}(m)]\left[\mathbf{v} \mathbf{v}^{1,1}(t+m, n, \beta)\right] \\
& +\left[\left[[\mathbf{P} \diamond \mathbf{H}(n-t)] \diamond \mathbf{C} \mathbf{K}_{1}(t, n-t, \beta)\right] \mathbf{1}^{\prime}\right] \diamond \mathbf{c}_{1}(t, n-1, \beta),
\end{aligned}
$$

with initial condition $\mathbf{v} \mathbf{v}^{1,1}(n-1, n)=\mathbf{0}$.

Equation 2.11 can also be expressed in closed analytic form by following the methodology of Papadopoulou (2004). Finally, from Eqs. 2.10 and 2.11 the variance of the total cost spent at time $n-1$ can be evaluated:

$$
\begin{aligned}
\operatorname{Var} & \left(\sum_{i=1}^{k} \sum_{t=0}^{n-1} R_{i}(t)\left(T_{t, n, \beta}^{i}-T_{t, n-1, \beta}^{i}\right)\right) \\
& =\sum_{t=0}^{n-1}(\boldsymbol{\Lambda}+(\mathbf{\Lambda} \diamond \mathbf{\Lambda}))\left(\mathbf{v}^{2}(t, n, \beta)+\mathbf{v}^{2}(t, n-1, \beta)-2 \mathbf{v} \mathbf{v}^{1,1}(t, n, \beta)\right) \\
& +\sum_{\left(i, t_{1}\right) \neq\left(j, t_{2}\right)} \lambda_{i} \lambda_{j}\left(v_{i}\left(t_{1}, n, \beta\right)-v_{i}\left(t_{1}, n-1, \beta\right)\right)\left(v_{j}\left(t_{2}, n, \beta\right)-v_{j}\left(t_{2}, n-1, \beta\right)\right) \\
& -\left(\sum_{t=0}^{n-1} \boldsymbol{\Lambda}(\mathbf{v}(t, n, \beta)-\mathbf{v}(t, n-1, \beta))\right)^{2},
\end{aligned}
$$

where $\mathbf{v} \mathbf{v}^{1,1}(t, n, \beta)$ is given in Eq. 2.11. 
2.3 System's Mean and Variance of the Total Cost Through the Interval $(0, \mathrm{n}]$ with Discounting

We have previously defined $T_{t, n, \beta}^{i}$ as the present value of cost spent by the system for a member until time $\mathrm{n}$, given that the member is recruited to state $\mathrm{i}$ at time $\mathrm{t}$. Then the present value of the total cost for all members of the system through the interval $(0, n]$ is equal to $\sum_{t=0}^{n} \sum_{i=1}^{k} R_{i}(t) T_{t, n, \beta}^{i}$.

Theorem 2.7 The mean of the present value of the total cost for all members of the system through the interval $(0, n]$ is equal to

$$
E\left(\sum_{t=0}^{n} \sum_{i=1}^{k} R_{i}(t) T_{t, n, \beta}^{i}\right)=\sum_{t=0}^{n} \boldsymbol{\Lambda} \mathbf{v}(t, n, \beta)
$$

where

$$
\begin{aligned}
\boldsymbol{\Lambda}= & {\left[\lambda_{1}, \lambda_{2}, \ldots, \lambda_{k}\right], \mathbf{v}(n, n, \beta)=\mathbf{0}, } \\
\mathbf{v}(t, n, \beta)= & \left(\mathbf{G}(n-t) \mathbf{1}^{\prime}\right) \diamond \mathbf{c}_{1}(t, n, \beta)+\mathbf{b}_{1}(t, n-t, \beta) \\
& +\sum_{j=2}^{n-t}[\mathbf{E}(j-1)]\left[\left(\mathbf{G}(n-t-j+1) \mathbf{1}^{\prime}\right) \diamond \mathbf{c}_{1}(t+j-1, n, \beta)\right. \\
& \left.+\mathbf{b}_{1}(t+j-1, n-t-j+1, \beta)\right], \\
\mathbf{G}(n)= & \operatorname{diag}\left\{\sum_{j=1}^{k} p_{1 j} \sum_{m=n+1}^{\infty} h_{1 j}(m), \ldots, \sum_{j=1}^{k} p_{k j} \sum_{m=n+1}^{\infty} h_{k j}(m)\right\}, \\
\mathbf{c}_{1}(t, n, \beta)= & {\left[(1-\beta)^{-1}\left(\beta^{t}-\beta^{n}\right) c_{1}, \ldots,(1-\beta)^{-1}\left(\beta^{t}-\beta^{n}\right) c_{k}\right]^{\prime}, } \\
\mathbf{b}_{1}(t, n, \beta)= & \sum_{m=1}^{n}\left[[\mathbf{P} \diamond \mathbf{H}(m)] \diamond \mathbf{C} \mathbf{K}_{\mathbf{1}}(t, m, \beta)\right] \mathbf{1}^{\prime} \\
& \left\{\sum_{j=1}^{k} p_{i j} \sum_{m=1}^{n} h_{i j}(m)\left[(1-\beta)^{-1} \beta^{t}\left(1-\beta^{m}\right) c_{i}+\beta^{t+m} k_{i j}\right]\right\}
\end{aligned}
$$

Proof Obviously, it is true that

$$
E\left(\sum_{t=0}^{n} \sum_{i=1}^{k} R_{i}(t) T_{t, n, \beta}^{i}\right)=\sum_{t=0}^{n} \sum_{i=1}^{k} \lambda_{i} E\left(T_{t, n, \beta}^{i}\right)=\sum_{t=0}^{n} \sum_{i=1}^{k} \lambda_{i} v_{i}(t, n, \beta)=\sum_{t=0}^{n} \boldsymbol{\Lambda} \mathbf{v}(t, n, \beta) .
$$


Theorem 2.8 The variance of the total cost for all members of the system through the interval $(0, n]$ is equal to

$$
\begin{aligned}
\operatorname{Var}\left(\sum_{t=0}^{n} \sum_{i=1}^{k} \lambda_{i} T_{t, n, \beta}^{i}\right)= & \sum_{t=0}^{n}(\boldsymbol{\Lambda} \diamond \boldsymbol{\Lambda}) \mathbf{v}^{2}(t, n, \beta)-\left(\sum_{t=0}^{n} \boldsymbol{\Lambda} \mathbf{v}(t, n, \beta)\right)^{2} \\
& +\sum_{\left(i, t_{1}\right) \neq\left(j, t_{2}\right)} 2 \lambda_{i} \lambda_{j} v_{i}\left(t_{1}, n, \beta\right) v_{j}\left(t_{2}, n, \beta\right)
\end{aligned}
$$

where

$$
\begin{aligned}
\boldsymbol{\Lambda}= & {\left[\lambda_{1}, \lambda_{2}, \ldots, \lambda_{k}\right], \mathbf{v}(n, n, \beta)=\mathbf{0}, } \\
\mathbf{v}(t, n, \beta)= & \left(\mathbf{G}(n-t) \mathbf{1}^{\prime}\right) \diamond \mathbf{c}_{1}(t, n, \beta)+\mathbf{b}_{1}(t, n-t, \beta) \\
& +\sum_{j=2}^{n-t}[\mathbf{E}(j-1)]\left[\left(\mathbf{G}(n-t-j+1) \mathbf{1}^{\prime}\right) \diamond \mathbf{c}_{1}(t+j-1, n, \beta)\right. \\
& \left.+\mathbf{b}_{1}(t+j-1, n-t-j+1, \beta)\right],
\end{aligned}
$$

where

$$
\mathbf{v}^{2}(t, n, \beta)=\mathbf{D}(t, n, \beta)+\sum_{j=2}^{n-t}[\mathbf{E}(j-1)][(\mathbf{D}(t+j-1, n, \beta)]
$$

and

$$
\begin{aligned}
& \mathbf{D}(t, n, \beta)=\left(\mathbf{G}(n-t) \mathbf{1}^{\prime}\right) \diamond \mathbf{c}_{2}(t, n, \beta)+\mathbf{b}_{2}(t, n-t, \beta) \\
& +2 \sum_{m=1}^{n-t}\left[[\mathbf{P} \diamond \mathbf{H}(m)] \diamond \mathbf{C K}_{1}(t, m, \beta)\right] \\
& \times\left[\left(\mathbf{G}(n-t-m) \mathbf{1}^{\prime}\right) \diamond \mathbf{c}_{1}(t+m, n, \beta)+\mathbf{b}_{1}(t+m, n-t-m, \beta)+\sum_{j=2}^{n-t-m}[\mathbf{E}(j-1)]\right. \\
& \times\left[\left(\mathbf{G}(n-t-m-j+1) \mathbf{1}^{\prime}\right) \diamond \mathbf{c}_{1}(t+m+j-1, n, \beta)\right. \\
& \left.\left.+\mathbf{b}_{1}(t+m+j-1, n-t-m-j+1, \beta)\right]\right] \text {, } \\
& \mathbf{G}(n)=\operatorname{diag}\left\{\sum_{j=1}^{k} p_{1 j} \sum_{m=n+1}^{\infty} h_{1 j}(m), \ldots, \sum_{j=1}^{k} p_{k j} \sum_{m=n+1}^{\infty} h_{k j}(m)\right\}, \\
& \mathbf{c}_{1}(t, n, \beta)=\left[(1-\beta)^{-1}\left(\beta^{t}-\beta^{n}\right) c_{1}, \ldots,(1-\beta)^{-1}\left(\beta^{t}-\beta^{n}\right) c_{k}\right]^{\prime} \text {, } \\
& \mathbf{b}_{1}(t, n, \beta)=\sum_{m=1}^{n}\left[[\mathbf{P} \diamond \mathbf{H}(m)] \diamond \mathbf{C K}_{1}(t, m, \beta)\right] \mathbf{1}^{\prime} \\
& =\left\{\sum_{j=1}^{k} p_{i j} \sum_{m=1}^{n} h_{i j}(m)\left[(1-\beta)^{-1} \beta^{t}\left(1-\beta^{m}\right) c_{i}+\beta^{t+m} k_{i j}\right]\right\}_{i=1,2, \ldots k},
\end{aligned}
$$


$\mathbf{C K}_{x}(t, m, \beta)$ is a $k \times k$ matrix, $\mathbf{C K}_{x}(t, m, \beta)=\left\{\left((1-\beta)^{-1} \beta^{t}\left(1-\beta^{m}\right) c_{i}+\beta^{t+m} k_{i j}\right)^{x}\right\}$ for every $x=1,2, \ldots$, and $\mathbf{C K}_{0}(m)=\mathbf{U}, \mathbf{c}_{2}(t, n, \beta)$ is a $k \times 1$ vector, where $\mathbf{c}_{2}(t, n, \beta)=$ $\left\{c_{\mathrm{i}}^{2}\left[(1-\beta)^{-1}\left(\beta^{t}-\beta^{n}\right)\right]^{2}\right\}, \mathbf{b}_{2}(t, n, \beta)$ is a $k \times 1$ vector and

$$
\begin{aligned}
\mathbf{b}_{2}(t, n, \beta) & =\sum_{m=1}^{n}\left[[\mathbf{P} \diamond \mathbf{H}(m)] \diamond \mathbf{C K}_{2}(t, m, \beta)\right] \mathbf{1}^{\prime} \\
& =\left\{\sum_{j=1}^{k} p_{i j} \sum_{m=1}^{n} h_{i j}(m)\left[(1-\beta)^{-1} \beta^{t}\left(1-\beta^{m}\right) c_{i}+\beta^{t+m} k_{i j}\right]^{2}\right\}_{i=1,2, \ldots k} .
\end{aligned}
$$

Proof We have that

$$
\begin{aligned}
& \operatorname{Var}\left(\sum_{t=0}^{n} \sum_{i=1}^{k} R_{i}(t) T_{t, n, \beta}^{i}\right)=E\left(\left(\sum_{t=0}^{n} \sum_{i=1}^{k} R_{i}(t) T_{t, n, \beta}^{i}\right)^{2}\right)-\left(E\left(\sum_{t=0}^{n} \sum_{i=1}^{k} R_{i}(t) T_{t, n, \beta}^{i}\right)\right)^{2} \\
& =E\left(\sum_{t=0}^{n} \sum_{i=1}^{k}\left(R_{i}(t) T_{t, n, \beta}^{i}\right)^{2}+\sum_{\left(i, t_{1}\right) \neq\left(j, t_{2}\right)} R_{i}(t) R_{j}(t) T_{t_{1}, n, \beta}^{i} T_{t_{2}, n, \beta}^{j}\right) \\
& -\left(\sum_{t=0}^{n} \sum_{i=1}^{k} \lambda_{i} E\left(T_{t, n, \beta}^{i}\right)\right)^{2} \\
& =\sum_{t=0}^{n} \sum_{i=1}^{k}\left(\lambda_{i}+\lambda_{i}^{2}\right) E\left(\left(T_{t, n, \beta}^{i}\right)^{2}\right)+\sum_{\left(i, t_{1}\right) \neq\left(j, t_{2}\right)} \lambda_{i} \lambda_{j} E\left(T_{t_{1}, n, \beta}^{i} T_{t_{2}, n, \beta}^{j}\right) \\
& -\left(\sum_{t=0}^{n} \sum_{i=1}^{k} \lambda_{i} v_{i}(t, n, \beta)\right)^{2} \\
& =\sum_{t=0}^{n} \sum_{i=1}^{k}\left(\lambda_{i}+\lambda_{i}^{2}\right) v_{i}^{2}(t, n, \beta)+\sum_{\left(i, t_{1}\right) \neq\left(j, t_{2}\right)} \lambda_{i} \lambda_{j} E\left(T_{t_{1}, n, \beta}^{i}\right) E\left(T_{t_{2}, n, \beta}^{j}\right) \\
& -\left(\sum_{t=0}^{n} \sum_{i=1}^{k} \lambda_{i} v_{i}(t, n, \beta)\right)^{2} \\
& =\sum_{t=0}^{n} \sum_{i=1}^{k}\left(\lambda_{i}+\lambda_{i}^{2}\right) v_{i}^{2}(t, n, \beta)+\sum_{\left(i, t_{1}\right) \neq\left(j, t_{2}\right)} \lambda_{i} \lambda_{j} v_{i}\left(t_{1}, n, \beta\right) v_{j}\left(t_{2}, n, \beta\right) \\
& -\left(\sum_{t=0}^{n} \sum_{i=1}^{k} \lambda_{i} v_{i}(t, n, \beta)\right)^{2} .
\end{aligned}
$$


The above result can equivalently be written as

$$
\begin{aligned}
\operatorname{Var}\left(\sum_{t=0}^{n} \sum_{i=1}^{k} R_{i}(t) T_{t, n, \beta}^{i}\right)= & \sum_{t=0}^{n}(\boldsymbol{\Lambda}+(\mathbf{\Lambda} \diamond \mathbf{\Lambda})) \mathbf{v}^{2}(t, n, \beta) \\
& -\left(\sum_{t=0}^{n} \boldsymbol{\Lambda} \mathbf{v}(t, n, \beta)\right)^{2}+\sum_{\left(i, t_{1}\right) \neq\left(j, t_{2}\right)} \lambda_{i} \lambda_{j} v_{i}\left(t_{1}, n, \beta\right) v_{j}\left(t_{2}, n, \beta\right) .
\end{aligned}
$$

\section{An Illustrative Example: Costing Stroke Patients}

We here use an open semi-Markov model with fixed size for costing thrombolysis services within a hospital (Diagram 1) where it is assumed that the hospital is very busy, so discharged patients are immediately replaced. Under this condition the open semi Markov model with fixed size can be considered as a closed semi Markov system (Vassiliou and Papadopoulou 1992). We notionally assign a oneoff cost to thrombolysis and daily costs to acute and rehabilitative care within the hospital. The model can then be used to determine means and variances of individual and total hospital costs within a given time period. Patients are initially admitted to thrombolysis, with probability $\theta$ or non-thrombolysis, with probability $1-\theta$. A thrombolysis patient is admitted to state $S_{1}$ where thrombolysis occurs; after a fixed duration (time taken for the therapy) these patients move into state $S_{2}$, postthrombolysis acute care. From there, they may be either discharged or move into a longer stay rehabilitative state $S_{3}$, from which they are eventually discharged. Nonthrombolysis patients initially move into an acute state $\mathrm{S}_{4}$ (acute care). Then, they may be discharged or move into a longer stay rehabilitative state $\mathrm{S}_{5}$. We assume daily occupancy costs in each of the five states and treatment cost of thrombolysis incurred in state $S_{1}$ and a transition cost from $S_{1}$ to $S_{2}$.

In modelling many operational systems the importance of discounting is almost negligible as costs are often essentially index-linked and are incurred on an ongoing basis. So, instead of the expected present values we can calculate costs without discounting i.e. $\beta=1$. In the present example we treat this special case.

Figure 1 presents the relationship between mean total cost, time and proportion of patients undergoing thrombolysis. Figure 2 shows mean daily costs (in GBP) for different proportions of patients, $\theta$, undergoing thrombolysis. Figure 3 presents the variance of the total cost for different proportions of patients undergoing thrombolysis. Figure 4 presents the change in mean total cost with respect to

Diagram 1 Patients pathways for a five state model of a stroke care unit

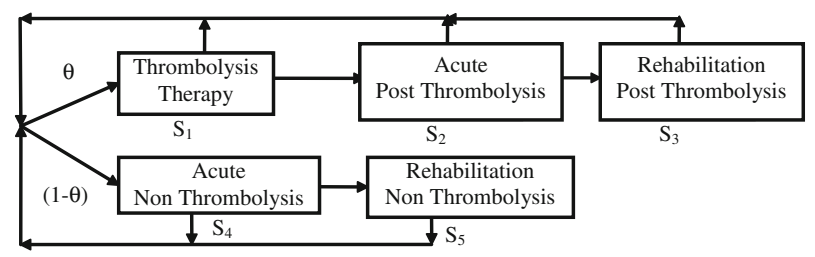


Fig. 1 Mean total cost for different proportions of patients having thrombolysis
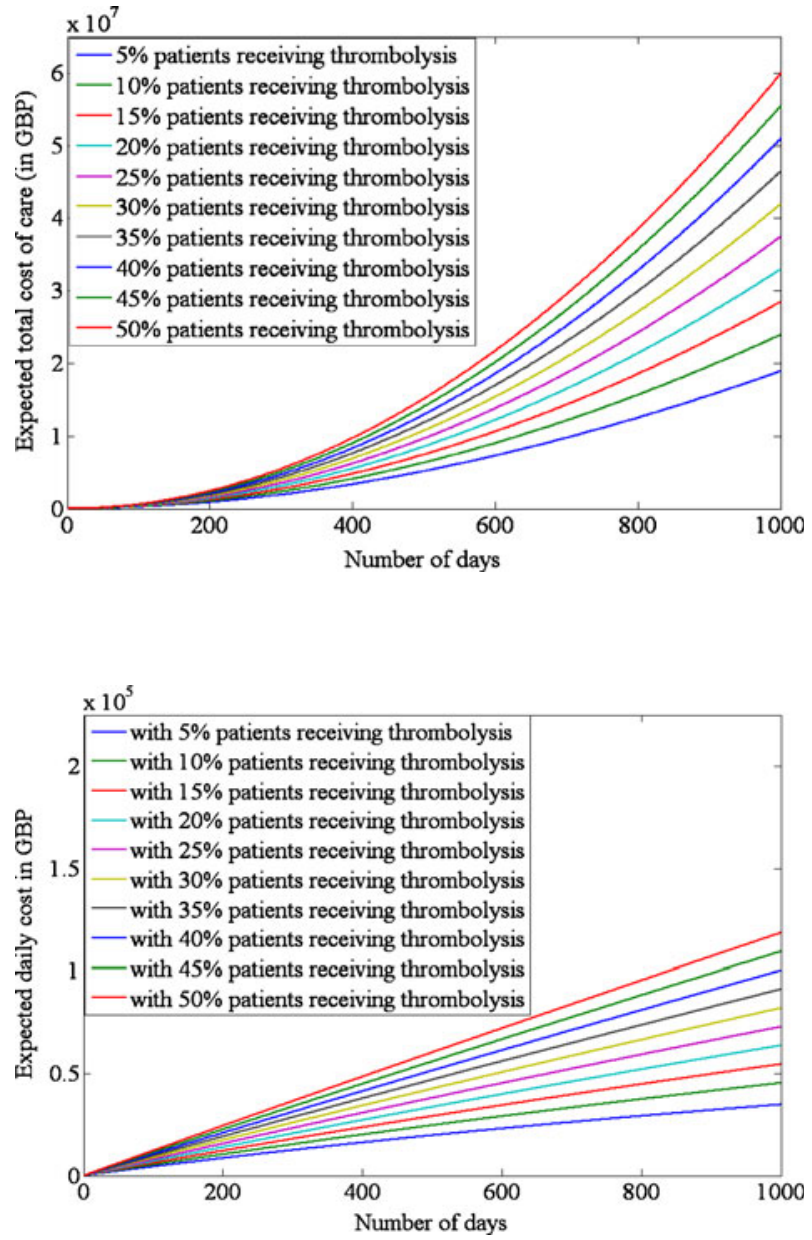

Fig. 2 Mean daily cost for different proportions of patients having thrombolysis

Fig. 3 Variance of the total cost for different proportions of patients undergoing thrombolysis

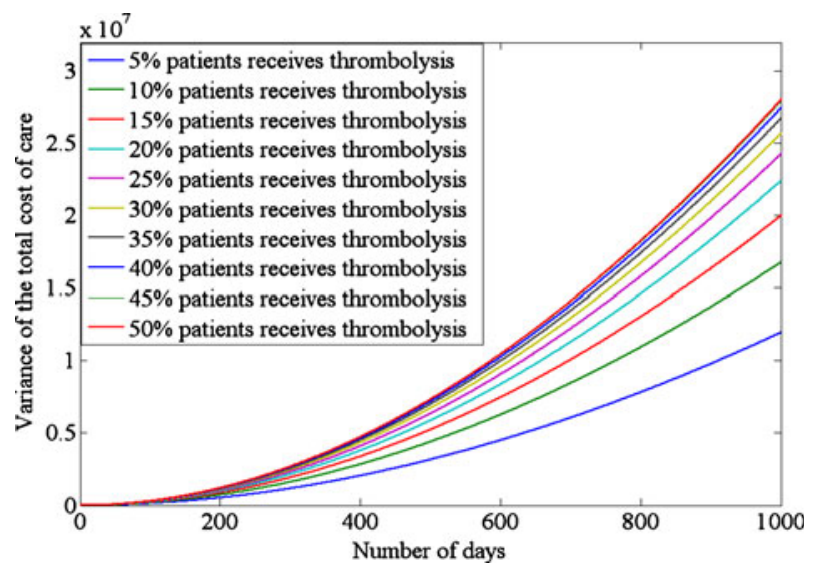


Fig. 4 Change in mean total cost and variance with change in duration if $10 \%$ patients $(\theta=0.1)$ have thrombolysis

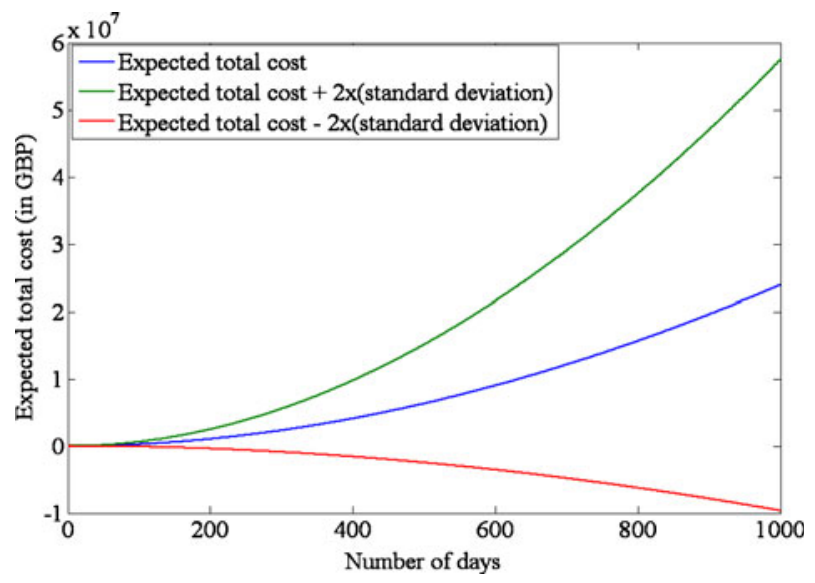

change in duration if $10 \%$ patients $(\theta=0.1)$ have thrombolysis. Figure 5 shows this change if $50 \%$ patients $(\theta=0.5)$ have thrombolysis. Figure 6 presents the plots of change in mean total cost with respect to change in the proportion of patients $(\theta)$ having thrombolysis. These figures also show values for mean total cost \pm 2 (standard deviation). The holding time probability mass functions are assumed to be geometric. The estimated matrix of the transition probabilities is given below

$$
\mathbf{P}=\left[\begin{array}{ccccc}
0 & 1 & 0 & 0 & 0 \\
0.331024 & 0 & 0.337953 & 0.331024 & 0 \\
0.5 & 0 & 0 & 0.5 & 0 \\
0.331024 & 0 & 0 & 0.331024 & 0.337953 \\
0.5 & 0 & 0 & 0.5 & 0
\end{array}\right]
$$

while the cost vector $\mathbf{c}=\left[\mathrm{c}_{1}, \ldots, \mathrm{c}_{5}\right]$ is given by $\mathbf{c}=[434.8,164.8,114.8,164.8,114.8]$, and $k_{12}=480, k_{i j}=0$ for $(i, j) \neq(1,2)$.

Fig. 5 Change in mean total cost and variance with change in duration if $50 \%$ patients $(\theta=0.5)$ have thrombolysis

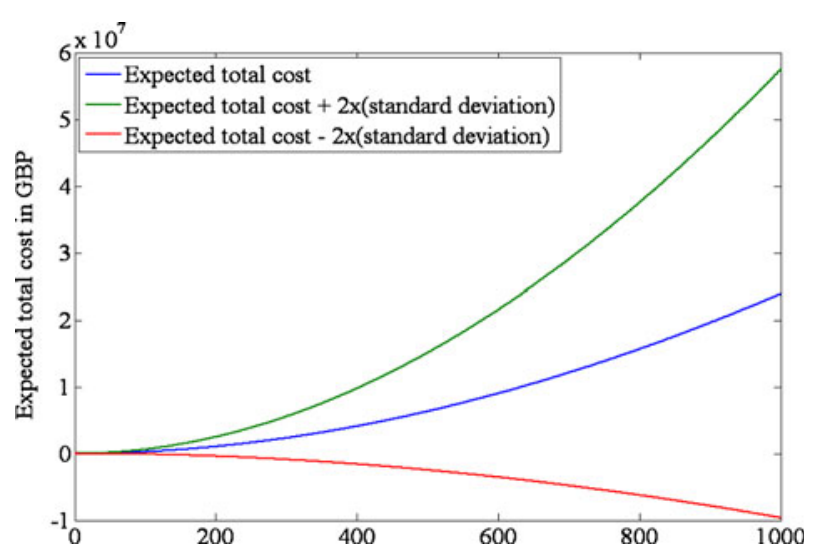


Fig. 6 Change in the mean total cost and variance with respect to the proportion of patients having thrombolysis (after 1000 days)

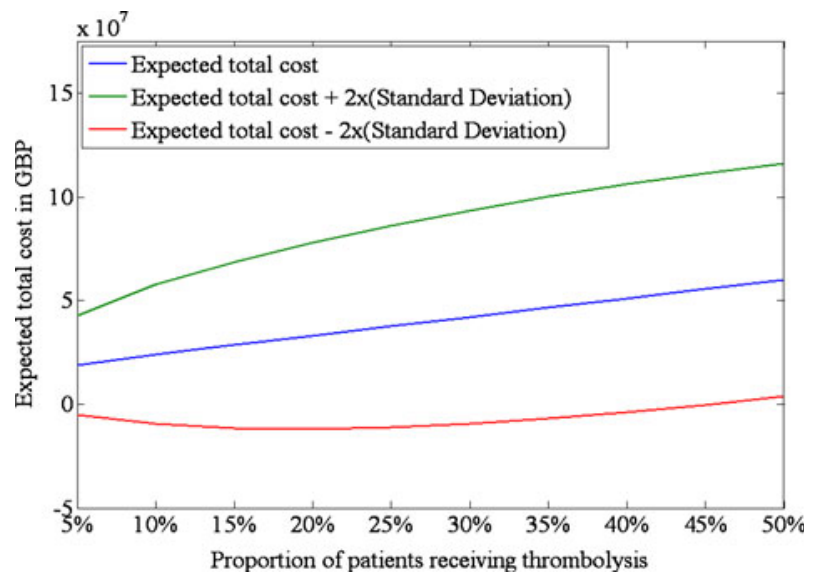

Apart from the positive values of the changes in mean total costs and variances appeared in Figs. 4, 5 and 6, it is obvious that the calculated negative values are essentially zero.

\section{Conclusions}

We have extended our previous work, which attached costs to duration in states for an open semi-Markov system by including costs of making a transition. Results have been obtained for the moments and the distribution of a member's cost as well as for the total costs to the system at any time. The resulting expressions have been applied to data for stroke patients, where costs are incurred per unit time in hospital and also for thrombolysis, which is a clot-busting therapy. The results demonstrate the potential of the models to demonstrate differential costs of various therapeutic strategies and explore optimal solutions.

Acknowledgements McClean would like to acknowledge support from the EPSRC (RIGHT and MATCH projects) and the NIHPSS R\&D Office (RIGHT project).

\section{References}

Balcer Y, Sahin I (1986) Pension accumulation as a semi-Markov reward process with applications to pension reform. In: Janssen J (ed) Semi-Markov models. Plenum, New York

De Dominicis R, Manca R (1986) Some new results on the transient behavior of semi-Markov reward processes. Methods Oper Res 54:387-397

Howard RA (1971) Dynamic probabilistic systems: semi-Markov and decision processes, vol II. Dover

Janssen J, Manca R (2007) Semi-Markov risk models for finance insurance and reliability. Springer

Jianyong L, Xiaobo Z (2004) On average reward semi-Markov decision processes with a general multichain structure. Math Oper Res 29(2):339-352

Limnios N, Oprisan G (2001) Semi-Markov processes and reliability. Birkhauser, Boston

Masuda Y (1993) Partially observable semi-Markov reward processes. J Appl Prob 30(3):548-560

Masuda Y, Sumita U (1991) A multivariate reward process defined on a semi-Markov process and its first-passage-time distributions. J Appl Prob 28(2):360-373 
McClean SI, Millard PH (2006) Where to treat the older patient? Can Markov models help us better understand the relationship between hospital and community care? J Oper Res Soc 58(2):255261

McClean SI, Papadopoulou A, Tsaklides G (2004) Discrete time reward models for homogeneous semi-Markov systems. Commun Stat, Theory Methods 33(3):623-638

McClean SI, Papadopoulou A, Tsaklides G, Garg L, Barton M, Millard PH (2008) Evaluating strategies using non-homogeneous Markov and semi-Markov systems. In: Proceedings of international workshop on applied probability, IWAP, Compiegne

Papadopoulou AA (2001) Rewards in a non-homogeneous semi Markov systems in discrete time. In: Proceedings of applied stochastic models and data analysis, Technical Univ. of Compiegne, France

Papadopoulou AA (2004) Economic rewards in non homogeneous semi-Markov systems. Commun Stat, Theory Methods 33(3):681-696

Papadopoulou A (2010) Modeling biological sequences and web navigation with a semi Markov chain. Proceedings of the Stochastic Modeling Techniques and Data Analysis International Conference, Chania, Greece, June 8-11, 2010

Papadopoulou AA, Tsaklidis G (2007) Some reward paths in semi Markov models with stochastic selection of the transition probabilities. Methodol Comput Appl Probab 9:399-411

Reza Soltani A, Khorshidian K (1998) Reward processes for semi-Markov processes asymptotic behaviour. J Appl Prob 35:833-842

Stenberg F, Manca R, Silvestrov D (2006) Semi Markov reward models for disability insurance. Theory Stoch Proces 12(28), no. 3-4:239-254

Stenberg F, Silvestrov D, Manca R (2007) An algorithmic approach to discrete time nonhomogeneous backward semi-Markov reward processes with an application to disability insurance. Methodology and Computing in Appl Probab 9:497-519

Vassiliou P-CG, Papadopoulou AA (1992) Non homogeneous semi-Markov systems and maintainability of the state sizes. J Appl Prob 27:756-76 